\title{
Role of Exosomes in Immune Microenvironment of Hepatocellular Carcinoma
}

\author{
Xiaojing Chen $\mathbb{D},{ }^{1}$ Hao Chi $\mathbb{D},,^{2}$ Xiaozhao Zhao $\left(\mathbb{D},{ }^{2}\right.$ Rui Pan $\left(\mathbb{D},{ }^{2}\right.$ Ying Wei $\mathbb{D},^{2}$ \\ and Yunwei Han (iD) \\ ${ }^{1}$ Department of Oncology, The Affiliated Hospital of Southwest Medical University, Luzhou, 646000 Sichuan, China \\ ${ }^{2}$ Clinical Medical College, Southwest Medical University, Luzhou, 646000 Sichuan, China \\ Correspondence should be addressed to Yunwei Han; hanyunwei@swmu.edu.cn
}

Received 5 December 2021; Accepted 8 January 2022; Published 28 January 2022

Academic Editor: Alessandro Granito

Copyright ( 12022 Xiaojing Chen et al. This is an open access article distributed under the Creative Commons Attribution License, which permits unrestricted use, distribution, and reproduction in any medium, provided the original work is properly cited.

Hepatocellular carcinoma (HCC) is the most common primary liver cancer. Since most patients with HCC are diagnosed at the intermediate or advanced stage and because HCC has a high incidence of metastasis and recurrence, it is one of the leading causes of cancer death. Exosomes are a subtype of extracellular vesicles and are typically $30-150 \mathrm{~nm}$ in diameter. Originating from endosomes, they can be secreted by almost all living cells. They are widely present in various body fluids and serve as an important medium for the interactions between cells. A series of studies have revealed that exosomes-mediated intercellular transfer of proteins, nucleic acids, and metabolites plays a crucial role in the initiation and progression of HCC, hypoxia and angiogenesis, chemotherapy sensitivity, and cell death mode and regulates the immune microenvironment. In this paper, we reviewed the recent researches on the multiple roles of tumor-associated exosomes in the progression of HCC. We laid particular focus on those researches that reveal how exosomes regulate the tumor immune microenvironment (TIME) and how exosomal cargos affect the progression of HCC. Besides, we emphasize some prospective directions to achieve a more accurate and complete analysis of the HCC immune microenvironment.

\section{Background}

Hepatocellular carcinoma (HCC) is the sixth most common cancer in the world and the third most fatal cancer [1]. Despite great breakthroughs in HCC treatments, such as surgical resection, radiotherapy, and immunotherapy, the prognosis remains poor. This is mainly because most HCC patients are diagnosed at the intermediate and advanced stages and lack specific biomarkers, therefore missing out on the optimal treatment window. Recently, attention has focused on immunotherapy for it greatly activates autoimmunity. Unfortunately, its therapeutic performance in clinical trials has been unsatisfactory. This is associated with the formation of the HCC immunosuppressive microenvironment which seriously hinders the antitumor effect of immune checkpoint inhibitors, attenuating the efficacy of immunotherapy [2]. At present, the mechanism for the formation and regulation of the immunosuppressive microenvironment remains unclear.
Exosomes are the smallest subtype of extracellular vesicles (EVs), ranging in diameter from 30 to $150 \mathrm{~nm}$ with a density of 1.13 to $1.19 \mathrm{~g} / \mathrm{ml}$ [3]. Regardless of the type or the physiological or pathological state of the originating cell, exosomes share common features as enriched with nucleic acids, lipids, and proteins [4]. In addition, the exosomes released from the same cell line can be highly homogeneous [5]. But the specific exosomal composition depends to some extent on the cell type and is also affected by different cell conditions or treatments. Compared with normal cells, tumor cells secrete more exosomes, and the number of exosomes secreted by different tumor cells varies dramatically [6]. And thanks to the lipid bilayer structure of exosomes, cargos contained in them are not easily degraded so as to convey cargos stably [7-9]. With the properties listed above, exosomal cargos have the potential to be used as biomarkers, therapeutic targets, or immunomodulators.

A number of studies have proved that exosomes are important mediators for the initiation, progression, 
angiogenesis, and metastasis of tumors [10]. Exosomes act as a signal-transmitting medium between cells to mediate intercellular communication and change the functional status of recipient cells [11]. In particular, the exosomal nucleic acids are usually accurately quantified after the exosomes are isolated, and their profiles can accurately reflect a variety of physiological conditions and diseases $[12,13]$. HCC-derived exosomes participate in changing tumor progression by regulating the tumor microenvironment and tumor immune status $[14,15]$. Therefore, the current research focuses on the interaction between exosomes and immune cells in HCC.

\section{Biogenesis and Acquisition of Exosomes}

2.1. Biogenesis and Accepting of Exosomes. Exosomes were first described in 1981 as ATPase-containing vesicles exfoliated from cells and were found in various normal cells and tumor cells [16]. The biogenesis of exosomes begins in the endosome. First, endocytic vesicles arise when the cellular membranes invaginate, and they fuse together to create early endosomes. After the early endosomes mature, they become late endosomes, namely, MVB. In the process, the early endosome membrane buds inwards and encapsulates the intracellular material, producing intraluminal vesicle (ILV) in the lumen of the organelle [17]. The biogenesis of exosomes mainly involves endosomal sorting complexes required for transport protein (ESCRT). ESCRT includes ESCRT-0, ESCRT-I, ESCRT-II, ESCRT-III, and related AAA-ATPase Vps4 complex [4]. Through its ubiquitin-interacting domain, ESCRT-0 drives the concentration and transfer of exosomal cargos and further recruits other ESCRT proteins, such as ESCRT-I, ESCRT-II, and ESCRT-III, to the endosomal membrane [18,19]. After the ESCRT complex is assembled, ESCRT-I and ESCRT-2 are responsible for the budding of endosomal membranes. Then ESCRT-III catalyzes the scission of necks of ILVs by forming polymer filament and finishing the inward budding $[20,21]$.

The sorting of exosomal cargos and the release of exosomes also require the involvement of apoptosis-linked gene-2 (ALG-2), interacting protein $X$ (Alix), tetraspanins (CD9, CD63, and CD81), and tumor susceptibility gene 101 protein (TSG101). Finally, the secretion of exosomes entails the participation of SNARE protein complexes and synaptic binding proteins. The MVB moves toward the cell surface, fuses with the cellular membrane, and afterwards releases the ILVs into the extracellular milieu as exosomes, which are then known as exosomes and enter into various body fluid environments [22,23]. In addition, studies have revealed the ESCRT-independent exosomal pathway, which depends on ceramide and tetrahydrocannabinol [24,25]. More notably, it is discovered that RAB31 has two roles in exosomes biogenesis: it drives the production of ILVs and protects MVB from degradation. The active RAB31, phosphorylated by multiple RTKs, interacts with flotillin proteins contained in lipid raft microdomains to induce the budding of the MVB membrane and then the formation of ILVs. Meanwhile, RAB31 attracts TBC1D2B to deactivate RAB7, inhibiting MVBs from merging with lysosomes and allowing ILVs to be secreted as exosomes [26].

The released exosomes travel through various body fluids and incorporate by recipient cells by several mechanisms, including direct membrane fusion, phagocytosis, micropinocytosis, receptor-ligand interaction, lipid raft mediated endocytosis, and clathrin or caveola coated pit [4] (Figure 1).

2.2. Acquisition of Exosomes from HCC Patients. Almost all cells can secrete exosomes [27] and exosomes are present in various body fluids. The exosomes in the blood can be divided into two major types: serum-derived exosomes and plasma-derived exosomes. Serum exosomal miR-320d [28], LUCAT1 [29], circAKT3 [30], circPTGR1 [31], etc. can be used as markers for the diagnosis and prognosis of HCC. Similarly, plasma exosomal miR-96 [32], lncRNA SENP3EIF4A [33], circUHRF1 [34], etc. can be used for the diagnosis of HCC as well. But it can be seen that serum exosomes and plasma exosomes as diagnostic molecular markers have certain differences in their composition. The main reason is that, in the process of obtaining serum, platelets in the blood coagulate and then secrete a large number of exosomes [35], which may cause interference in the test results. As a result of this potential interference, it is recommended to choose plasma samples unless the plateletrelated disease is the object of study. It is worth noting that the expression of the same molecule in serum exosomes and plasma exosomes may have different significances. Serum exosomal miR-21-5p is correlated with advanced stage and it is an independent prognostic factor for disease-free survival in patients with early HCC [36]. Meanwhile plasma exosomal miR-21-5p can be used as a potential biomarker for the diagnosis of HCC [37].

Although current studies of exosomes focus on bloodderived exosomes, we should not ignore the potential of exosomes from other body fluids (Figure 2). Actually, exosomes can be obtained from the urine and ascites of HCC patients. Exosomes from the urine of HCC patients were successfully extracted and it was found that LINC00161 was expressed in urine [38]. Furthermore, it has been found that the expression levels of ascitic fluid-derived exosomal miR182, miR-301a, and miR-373 were significantly upregulated in liver cancer [39]. As bile is a fluid directly secreted by liver cells, bile-derived exosomes might be more organ-specific and sensitive. Meanwhile, bile exosomal miR-483-5p and miR-126-3p were found to have diagnostic values for malignant biliary obstruction [40]. At present, there is little research related to bile-derived exosomes in HCC patients.

To date, tissue-derived exosomes show unique advantages. By using electron microscopy, researchers found that there are extracellular vesicles of different sizes in the extracellular space of prostate cancer tissues and melanoma tissues and thereby confirmed the existence of exosomes in the interstitial space $[41,42]$. These extracellular vesicles can reveal the current physiological and pathological conditions of the tissue and participate in the generation of tissue microenvironment [4]. Previously, exosomes from brain tissues digested by collagenase type 3 have been successfully 


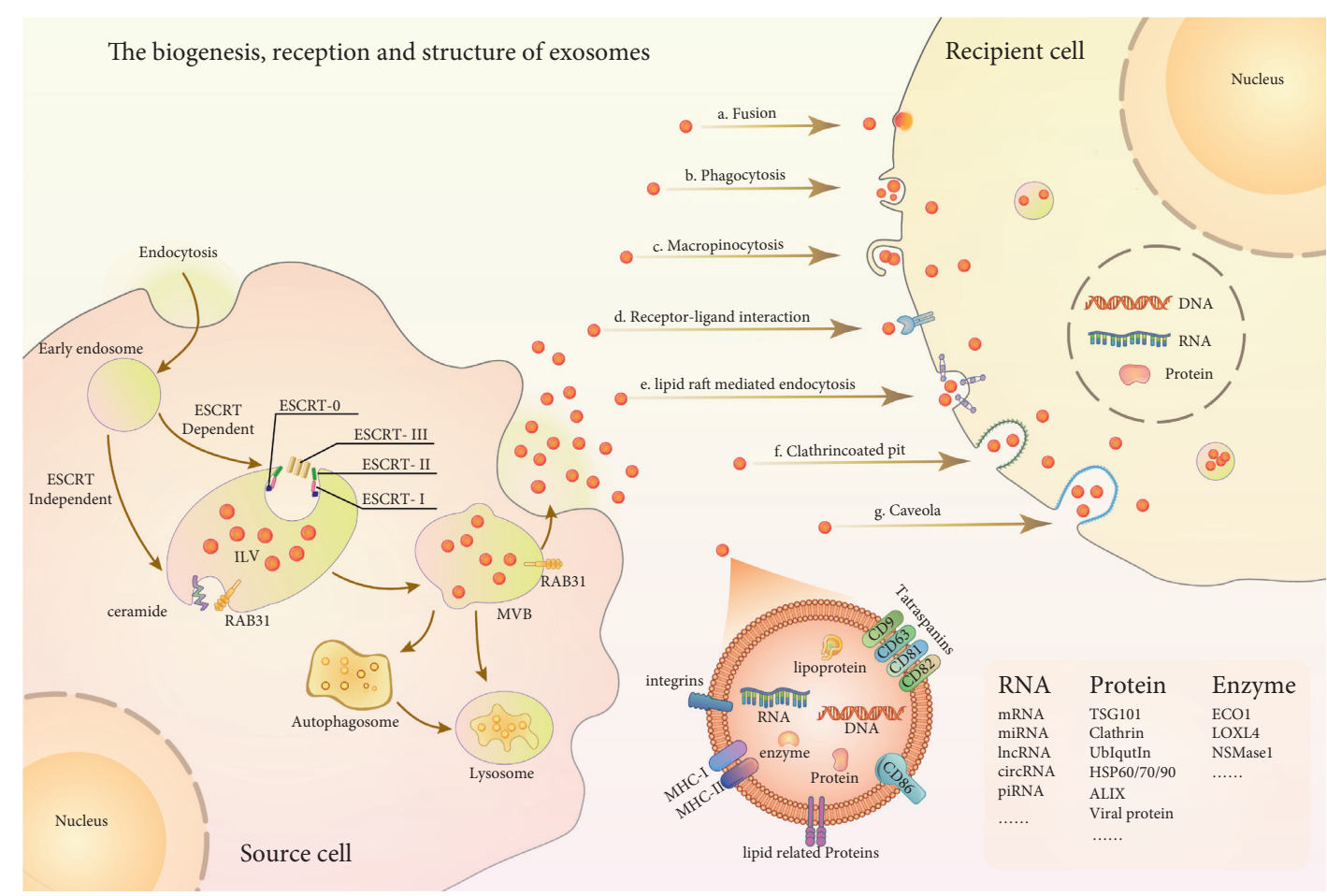

FIGURE 1: Schematic illustration of the biogenesis, secretion, absorption, and composition of exosomes. Exosome biogenesis initiates with the process of inward budding of the cell membrane, which is called endocytosis, followed by the formation of endosomes. Then the ILVs were formed by the invagination of the endocytic membrane into the organelle lumens; thus the endosomes turn into MVBs. The MVBs fuse with the plasma membrane through ESCRT-dependent or ESCRT-independent pathways to release exosomes, otherwise, fusing with lysosomes for degradation or ending up to autophagosomes. Subsequently, the released exosomes can be uptaken by recipient cells via fusion, phagocytosis, micropinocytosis, receptor-ligand interaction, lipid raft mediated endocytosis, and clathrin or caveola coated pit. Exosome contains an assortment of bioactive cargos, including RNA, protein, and enzyme.

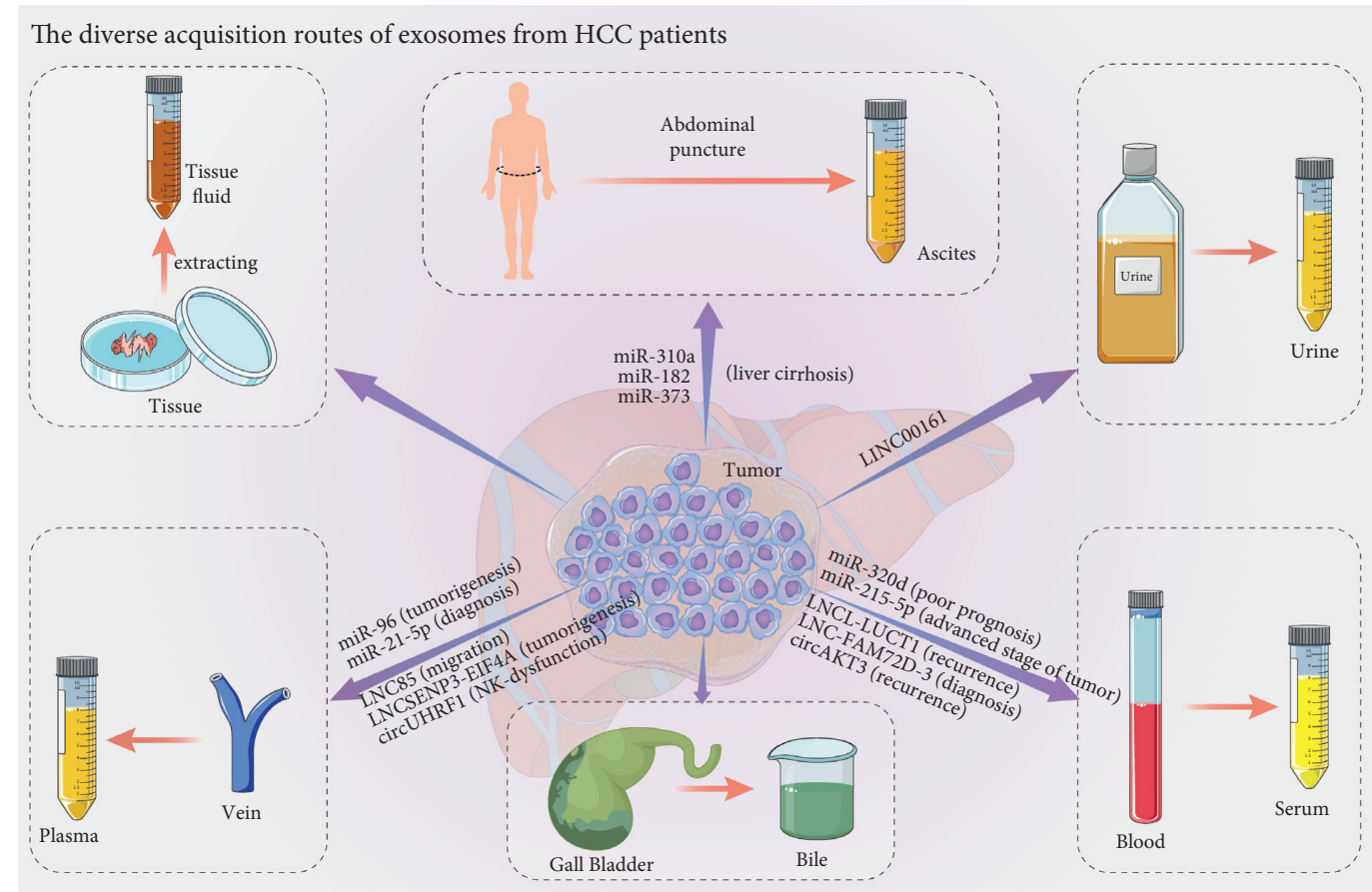

FIgURE 2: The diverse acquisition routes of exosomes from HCC patients. Exosomes participate in HCC progression and can be extracted from different sources. Exosomal cargos such as small RNA, long noncoding RNA, and circ RNA regulate the malignant phenotypes and can be used as markers for the diagnosis, prognosis, recurrence, migration, and invasion ability of HCC and tumor angiogenesis. 
extracted [43]. And then the protocol of enzymatic treatment of dissociated issues followed by differential ultracentrifugation and density separation was proposed. Through this approach, up to six extracellular vesicles subpopulations were isolated, including exosomes [44]. These methods showed that we can extract exosomes from tissues. Whereas exosomes in the blood are derived from multitissue cells, the exosomes secreted by the cells exist first and foremost in interstitial space, which means that tissue-derived exosomes can better reveal the tumor microenvironment. However, there are few studies on exosomes derived from HCC tissues, which leave room for further exploration.

\section{Exosomal Cargos as Biomarkers or Regulators in HCC}

3.1. Exosomal DNA. Thanks to the lipid bilayer membrane, which provides exosomes enough space to package relatively large molecular weight DNA and can serve as a highly sensitive and specific biomarker for tumors $[45,46]$. Similarly, cell-free DNA (cfDNA) is also considered as a potential diagnostic biomarker. But the detection rate of exosomal DNA in early HCC patients was higher than that of cfDNA [47], because early liver cancer cells derived exosomal DNA can be secreted into body fluids, while cfDNA is derived from damaged, apoptotic, or necrotic cancer cells and its level will not increase significantly until HCC progresses into advanced stage [48]. In addition, pancreatic ductal adenocarcinoma patients revealed that the KRAS mutation rate in exosomal DNA is significantly higher than that in cfDNA [49]. Thus, exosomal DNA may hold more potential for early HCC diagnosis with unique features.

3.2. Exosomal mRNA. Efforts are being made to explore the application of exosomal mRNA. The expression level of exosomal hnRNPH1 mRNA in HCC patients increases significantly and is positively correlated with portal vein tumor thrombosis, Child-Pugh score, TNM staging, and lymph node metastasis [50]. Similarly, the serum exosomal RAB11 A mRNA shows high specificity (73.3\%) and sensitivity $(75 \%)$ in distinguishing healthy people, chronic hepatitis C patients, and HCC patients [51]. Apparently, exosomal mRNA can be used as an independent HCC diagnostic marker.

3.3. Exosomal miRNA. A large number of studies have analyzed the genetic materials present in exosomes. Among them, the role of Exo-miRNA in HCC is by far the most widely described. Next, we introduce several in-depth studies of hypoxia, angiogenesis, epithelial-to-mesenchymal transition (EMT), chemotherapy sensitivity, etc. and discuss the various roles that exosomal miRNAs can play under different conditions.

3.3.1. Exosomal miRNA Is Involved in Regulating the Hypoxia. The diffusion limitation of oxygen and the anatomical or physiological abnormalities of tumor vasculature can cause varying degrees of hypoxia in solid tumors. Tumor cells around normal functional blood vessels are viable and proliferative, while solid tumors about $100 \mu \mathrm{m}$ away from functional blood vessels usually exhibit hypoxia [52]. And hypoxia is the dynamic and heterogeneous characteristic of most solid tumors [53], while hypoxic tumor cells integrate existing blood vessels nearby or form new blood vessels by inducing tumor angiogenesis factors, thereby establishing a new tumor microenvironment [54]. Under hypoxic conditions, tumor cells secrete a fairly great number of exosomes. Whereas hypoxia in normal cells leads to cell death, hypoxia in tumor cells can induce specific exosomes release to adapt to the unfavorable microenvironment (Figure 3). For example, exosomal miR-155, miR-378b, and circCMTM3 derived from HCC cells promote angiogenesis in human umbilical vein endothelial cells under hypoxic conditions [55-57]. Interestingly, HCCderived exosomal miR-3682-3p as a negative regulator of angiopoietin-1 inhibited endothelial cells resulting in angiogenesis impairment; consequently, tumor cells downregulated its expression [58].

Energy metabolism reprograms through exosomes and impels tumor cells to achieve malignant proliferation with hypoxia. Under hypoxic conditions, HCC cells promote the secretion of exosomal linc-RoR, which can activate the HIF- $1 \alpha$, thereby enhancing the glycolysis to resist the hypoxic environment [59]. Typically, glycolysis is a prominent feature of tumors under hypoxic conditions, while another major feature is the low PH caused by high levels of lactic acid [60]. Then the acidic microenvironment triggers the expression of miR-21 and miR-10b in the exosomes of HCC cells and promotes proliferation and metastasis [36]. In addition, exosomal cargos can also induce normoxic cells to establish malignant phenotypes. Hypoxia cells are induced to produce more exosomes and then promote the expression of miR-1273f in normoxic HCC cells targeting LHX6 for downregulation, thereby enhancing the EMT and metastasis of normoxic HCC cells [61].

\subsubsection{Exosomal miRNA Regulates the EMT Procession.} EMT is the process of epithelial cells transitioning into mesenchymal cells with migration and invasion properties, which are closely related to tumor metastasis [62]. Exosomes containing miRNA can induce EMT in HCC. Exosomal miR-24-3p produced after SMMC-7721 cells treated with transforming growth factor $\beta$ (TGF- $\beta$ ) undergo EMT can promote the migration and invasion of not treated SMC-7721 cells [63]. Besides, exosomal miRNA-451a derived from HUCMSCs can inhibit ADAM10 to decrease EMT in HCC [64]. At the same time, the exosomes derived from HCC cells can also mediate EMT through TGF- $\beta /$ Smad and MAPK/ERK signaling pathways $[65,66]$. Mesenchymal-epithelial transition (MET) is EMT in reverse and a large number of studies have shown that MET also plays an important role in the metastasis of breast cancer, pancreatic cancer, and other tumors [67]. Whether these roles are present in HCC, however, requires further research. 


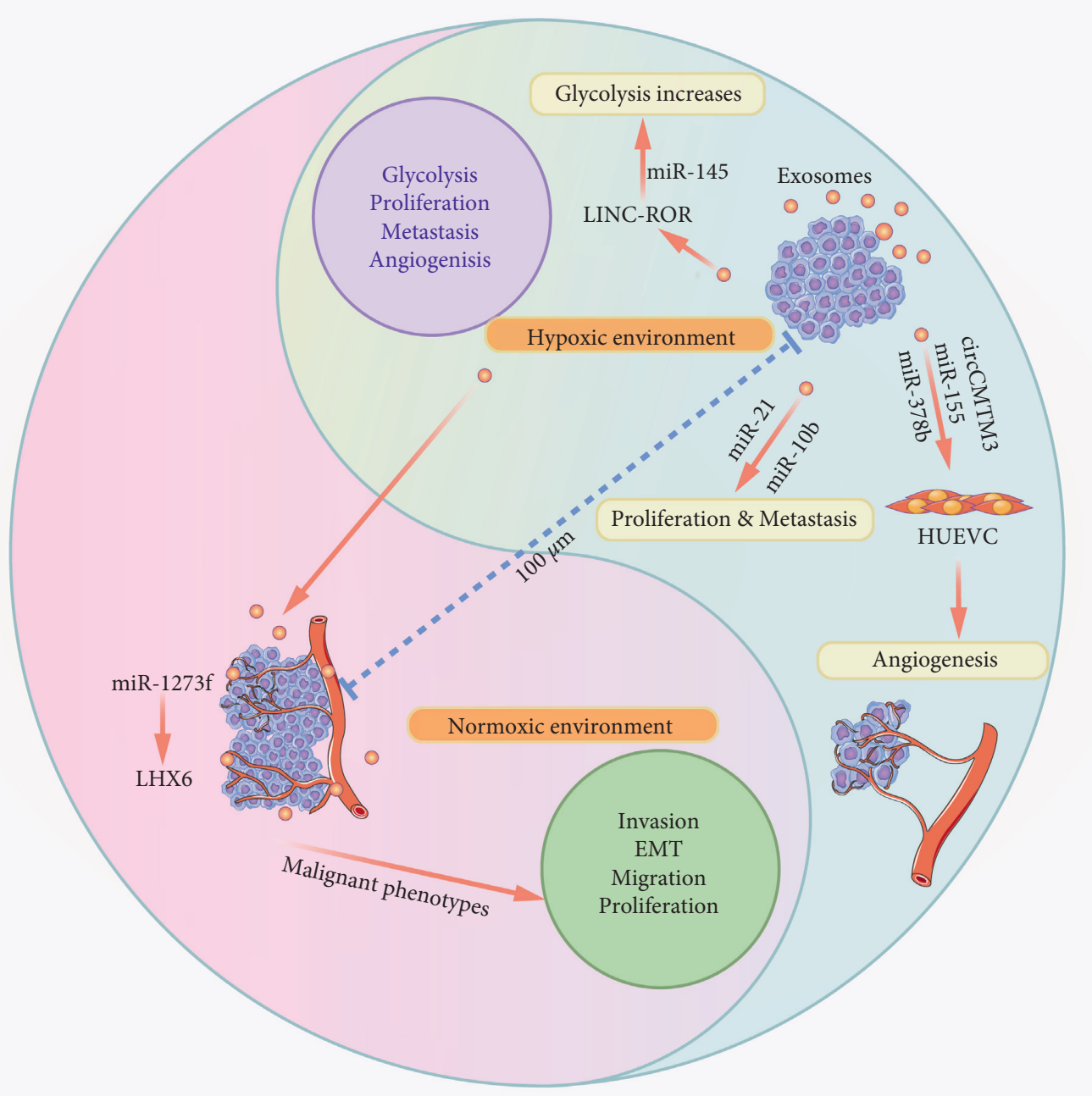

FIgURE 3: Exosomal miRNA is involved in regulating the hypoxic. Hypoxia usually occurs in solid tumors about $100 \mu \mathrm{m}$ from the functional vessels. Under hypoxia conditions, tumor cells activate multiple signaling pathways to enhance the release of specific cargos in exosomes and induce angiogenesis, glycolysis, self-proliferation, and metastasis, thereby adjusting their adaptability to hypoxia environment. Additionally, they promote the malignant phenotype of normoxic tumor cells and lead to disease progression.

\subsubsection{Exosomal miRNA Regulates the Chemotherapy} Sensitivity. To date, HCC shows high resistance to commonly used chemotherapy drugs such as fluorouracil, doxorubicin, and cisplatin [68]. Recent studies have shown that exosomal miRNAs are related to cancer chemosensitivity [69, 70]. Exosomal miR-451a can increase the chemosensitivity of HCC to paclitaxel by targeting ADAM10 [64]. Similarly, miR-199a can modify the exosomes from adipose-derived mesenchymal stem cells and thereafter increase the sensitivity of HCC cells to doxorubicin via the mTOR pathway [71]. Exosomes from miR-122-modified adipose-derived mesenchymal stem cells (AMSCs) can inhibit the expression of ADAM10, IGF1R, and CCNG1, thereby increasing the chemosensitivity of HCC cells to sorafenib [72]. Analogously, exosomal miR-744 inhibits the proliferation of HCC cells by targeting PAX2 and increases the sensitivity to sorafenib [73]. Furthermore, exosomes derived from human embryonic kidney epithelial cells function as nanoparticles to transfer miR-199a-3p, thereby reversing the chemoresistance of HCC cells to cisplatin [74].

In addition to improving the sensitivity of HCC to chemotherapy drugs, exosomal miRNAs can also increase drug resistance. For example, adipose-derived exosomal miR-23a and miR-23b confer HCC cells with chemoresistance to fluorouracil [75]. The tumor suppressor gene PTEN is often associated with acquired resistance to drugs [76, 77]. The exosomal miR-32-5p derived from multidrugresistant HCC cells inhibits PTEN and activates the PI3K/ Akt pathway, thereby inducing multidrug resistance further to transform sensitive cells into resistant cells [78].

In short, exosomes can mediate the chemotherapy sensitivity or resistance of tumor cells through miRNAs. Meanwhile, the above evidence also indicates that many exosomes derived from normal cells are vital players in regulatory role in the chemotherapeutic sensitivity of HCC. As a result, the exosomes of normal cells, functioning as 
delivery vehicles to improve the sensitivity of HCC chemotherapy which could be a new strategy to inhibit HCC, are worthy of our attention.

3.3.4. Exosomal miRNA Regulates the Cell Death Methods. Some researchers divide the cell death methods roughly into two types: accidental cell death (ACD) and regulated cell death (RCD). According to the morphological, biochemical, and genetic characteristics of various cell death modes, apoptosis, autophagy, pyrolysis, and ferroptosis can be classified as RCD and necrosis as ACD [79]. The extensive role of exosomal miRNA has been shown in a variety of cell death mechanisms, which consist of apoptosis, autophagy, pyroptosis, ferroptosis, and necroptosis [80-82].

In normal conditions, apoptosis, which helps to maintain the relative balance between cell death and regeneration, depended on the Caspase and BCL-2 family [80]. Resistance to apoptosis and maintenance of cell growth are the two main characteristics of cancer. The same HCC-derived exosomes can increase the expression of miR-21 and p-Akt in HCC cells and decrease the expression of PTEN and TETs, which inhibits cellular apoptosis [83].

Autophagy is a process of cell's self-digestion and catabolism, which degrades organelles and other subcellular structures via lysosomes and recycles the degraded products $[84,85]$. Previous experimental studies demonstrated that autophagy has a dual role in the progression of HCC [86]. It is shown that HCC-derived exosomal miR-3091-3p can inhibit the expression of Atg9b in liver cells, thereby inhibiting autophagosome-lysosome degradation [87].

Ferroptosis, resulting from iron-dependent lipid peroxide accumulation, is involved in tumor progression as a new type of RCD [88]. Exosomal miR-522 secreted by cancer associated fibroblasts inhibits ferroptosis of gastric cancer cells by targeting ALOX15 and blocking lipid-ROS accumulation [69]. HEK-293 T cells derived exosomes loaded with CD47 and ferroptosis inducer erastin can significantly promote ferroptosis of HCC cells [89]. However, whether exosomal miRNAs can participate in the ferroptosis of HCC is worthy of further research.

Evidence showed that pyroptosis can regulate tumor progression. In gastric cancer, ADAMTS9-AS2 serves a tumor-inhibiting role by activating NLRP3-mediated cell pyroptosis through sponging miR-223-3p [90]. But the relationship between necrosis and exosomes has not been studied sufficiently in the field of HCC. Interestingly, EVencapsulated miR-379 derived from mesenchymal stem cells to target breast cancer cells exhibits greater areas of central necrosis [91].

The purpose of tumor treatment is to inhibit and eliminate tumor cells. And exosomes participate in these varieties of death modes by influencing related genes and molecules upstream. However, how exosomes function in these mechanisms requires further exploration. Presumably, engineered exosomes for targeted drug delivery can be used to induce cell death with high selectivity and without affecting the life cycle of normal cells.
3.4. Exosomal lncRNA and circRNA. Exosomes are also known to contain IncRNA and circRNA, which can function as competitive endogenous RNA. These noncoding RNAs packaged in exosomes have been recently proved to spongeabsorb miRNAs and then regulate mRNA stability and translation so as to alter HCC progression. Particularly, it contributes to HCC cells' malignant phenotypes with proliferation, migration, invasion, EMT: circ-DB [92], circCdr1as [93], circ-0072088 [94], lnc85 [95], C5orf66-AS1 [96], DLX6-AS1 [97], or circ-004277 [98]. Others have been proved associated with HCC development such as LINC00511 [99], HOTAIR [100], circ-0070396 [101], CircWHSC1 [102], or circ-0074854 [103]. Besides, exosomal lncRNA and circRNA are supposed to be more sensitive and specify biomarkers than Alpha-fetoprotein (AFP). According to the differential expression of serum lnc85, AFP-negative HCC patients can be distinguished from cirrhosis and healthy control group [95]. Similarly, exosomal circ0070396 is superior to AFP in distinguishing HCC patients from healthy persons and can also be used to distinguish liver cancer patients from patients with chronic hepatitis B and patients with cirrhosis [101].

3.5. Exosomal Protein. Previously, it has been confirmed that exosomes from HCC cells contain thousands of proteins, which can be endocytosed by surrounding adipocytes, thereby creating a tumor microenvironment conducive to the progression of HCC [50]. Later, evidence showed that exosomal protein plays a strong regulatory role in the formation of the tumor microenvironment. Exosomal p120catenin can inhibit the metastasis and proliferation of HCC stem cells by activating the STAT3 pathway [104]. Exosomal S100A4 is a key enhancer by upregulating OPN expression through the STAT3 signaling pathway for HCC metastasis [105]. Besides, exosomal proteins are involved in mediating the immune escape of HCC. Tumor-derived exosomal HMGB1 can expand regulatory B cells by upregulating TIM1 and thereby promote the immune escape of HCC cells [106]. Yet there are still many gaps in the field of exosomal proteins in HCC, which need further exploration.

3.6. Exosomal Enzyme. Enzymes also can be delivered by exosomes to regulate HCC development. The amount and activity of the exosomal NSNase1 (neutral sphingomyelinase 1) isolated from HCC tissues are lower than normal tissues, resulting in a higher sphingomyelin/ceramide ratio and then inhibiting apoptosis and promoting the growth in HCC [107]. ENO1 can be transferred from highly metastatic HCC cells to low-metastatic-potential HCC cells through exosomes, which promote the growth and metastasis of HCC cells with low ENO1 expression by upregulating integrin $\alpha 6 \beta 4$ expression and activating the FAK/Src-p38MAPK pathway [108].

3.7. Exosomal Lipids or Other Metabolites. Metabolites such as lipids, amino acids, and inorganic substances in exosomes are involved in tumor regulations [109]. In exosomes 
secreted by Huh7 liver cancer cells, cardiolipin, phosphatidylserine, phosphatidylglycerol, and lanosylinositol are all enriched [110]. Ten lipid classes identified from plasma exosomes were enriched in HCC exosomes compared with non-HCC exosomes and these changes reflected alterations in glycerophospholipid metabolism, retrograde endocannabinoid signaling, and ferroptosis [111]. Although many HCC-associated lipids and other metabolites were identified from exosomes, their roles have not been elucidated and require further clarification.

\section{Exosomes as TIME Regulators in HCC}

Tumor cells and their surroundings form a functional unit that can be split into two types: the immune and the nonimmune microenvironment characterized by immune cells and fibroblasts separately. In the TIME, tumor cells and immune cells communicate with each other through cell contact and secretion of soluble molecules and exosomes. Therein, exosome is one of the key factors that mediate the formation of immunosuppressive microenvironment (Figure 4). In HCC, immune cell-derived exosomes play a vital role in regulating the metastasis, invasion, and immune escape $[112,113]$. Therefore, in the following sections, we will elaborate the latest research progress on the mechanism of exosomes affecting various types of immune cells and explore the mutual information transmission mechanism of exosomes in the TIME of HCC.

4.1. Neutrophil. Neutrophils are subdivided into two subtypes according to their phenotype in the tumor microenvironment: antitumor type (N1) and protumor type (N2) [114]. The immunosuppressive TGF- $\beta$ can promote the N2 phenotype, and the interferon $\beta / \gamma$ (IFN $\beta / \gamma)$ can promote the N1 phenotype [115]. As reported in HCC, the synergistic effect of TGF- $\beta$ and Axl induces the infiltration of neutrophils into HCC tissue and promotes tumor progression [116]. HMGB1 plays a vital role in the neutrophil recruitment of acute liver injury in HCC [117]. Zhang pointed out that HMGB1 is enriched in gastric cancer-derived exosomes and can induce neutrophil autophagy and activate tumorpromoting effects [118]. But the mechanism of neutrophils phenotypes with exosomes in the shaping of the tumor microenvironment of HCC is still unclear.

4.2. Macrophage. The M0 macrophage can polarize to the M1 macrophage with immune surveillance effect or the M2 phenotype with a proinflammatory effect [119]. Although M1 macrophage is the cell type with a stronger capacity of phagocytosis and antigen presentation, M2 macrophage is the major phenotype in the tumor microenvironment and promotes tumor progression [120]. Multiple factors affect the polarization of macrophages, and exosomes serve as an important medium in the interaction between macrophages and HCC [121]. For example, HCC-derived exosomal TUC339 can increase the number of M2 macrophages upon treatment of IFN- $\gamma$ and LPS, thereby regulating M1/M2 macrophages polarization [122]. The upregulated miR-146a-
$5 p$ in exosomes secreted by HCC cells can drive T cell exhaustion through M2 macrophages [123].

Endoplasmic reticulum stress (ER stress), a common feature of tumor cells, can regulate tumor immunity by regulating the function of macrophages in the tumor microenvironment [124]. ER stress induced release of exosomal miR-23a-3p from HCC cells can upregulate the expression of PD-L1 in macrophages and then upregulated PD-L1 interacts with $\mathrm{T}$ cell $\mathrm{PD}-1$ and further facilitates tumor progression by inhibiting $\mathrm{T}$ cell proliferation [125]. In addition, macrophages produced exosomes directly in return to regulate HCC cells. Macrophage-derived exosomal miR92a-2-5p can increase metastasis of HCC cells by altering AR/PHLPP/p-AKT/ $\beta$-catenin signaling pathway [126]. Polarized M2 macrophages can promote metastasis by transferring $\mathrm{CD} 11 \mathrm{~b} / \mathrm{CD} 18$ integrins to HCC cells through exosomes [127].

Macrophages are the most numerous immune cells in the immune microenvironment. It might be a good direction in tumor treatment to enhance M1 macrophage polarization and reduce the number of M2 macrophages by using exosomes. In addition, due to the regulatory effect of exosomes in ER stress inducing the macrophages to switch into M2 macrophages, we should not ignore its important influence on tumor metastasis [128].

4.3. Dendritic Cell. Dendritic cell (DC) can take up and present tumor-associated antigens, while exosomes serve as transmission carriers in this process. In a subcutaneous tumor model, DCs loaded with tumor exosomes exhibited anticancer activity. The anti-HCC efficacy can be enhanced through microwave ablation technology combined with DC cell-derived exosomal vaccine [129]. DC-derived exosomes (DEX), which contain MHCI/MHCII/CD86/HSP70-90 chaperones, can trigger the activation of $\mathrm{CD}^{+}$and $\mathrm{CD}^{+}$ T cells [130]. In addition, DEX containing alpha-fetoprotein (AFP) can stimulate mice with HCC to produce more IFN- $\gamma$ and IL-2, which was conducted by the increase of $\mathrm{CD} 8^{+}$ $\mathrm{T}$ cells and then decreased the regulatory T cells (Tregs), IL10 , and TGF- $\beta$, improving the tumor microenvironment [131].

However, the immunosuppressive effect of exosomes and DC in the local microenvironment cannot be ignored. Tumor-derived exosomes can block the differentiation of myeloid precursor cells into DC and inhibit the differentiation of bone marrow DC cells, ultimately inducing the formation of DC immune tolerance [132]. Exposure to IL-6/ IL-10/PGE2/VEGF/TGF- $\beta$ or costimulatory inhibitory pathways can induce the increased proportion of tolerogenic DCs and the apoptosis of alloreactive T cells, and the abovementioned cytokines are expressed in the progression of HCC involving exosomes (especially TGF- $\beta$ and IL-10) [133].

In short, delving into the dual immune response of DC cells helps to reveal a more detailed DC immune landscape-that is, inducing the antitumor effect of alloreactive $T$ cells on the one hand and mediating the immune evasion HCC by inducing the tolerance to tumor antigen on the 


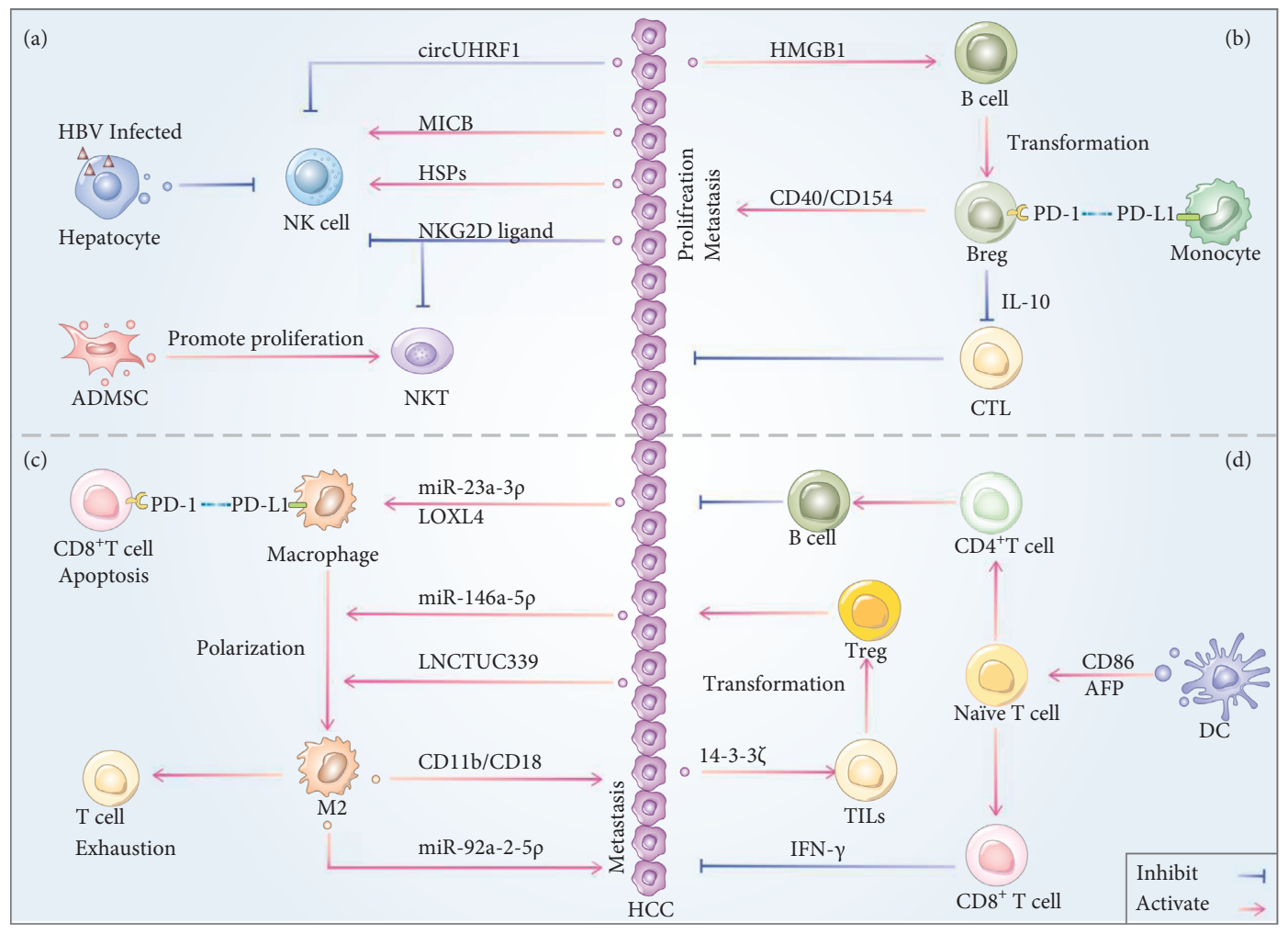

Figure 4: The landscape of tumor Immune microenvironment regulated by exosomes in HCC. (a) Exosomes containing specific cargos activate the function of NK cell or inhibit the function of both NK cell and NKT cell, while the function of NK cell can be inhibited by exosomes secreted by HBV infected hepatocyte and the proliferation of NKT cell can be boosted by exosomes from ADMSC. (b) Breg cells either release IL-10 to inhibit the function of CTL, which participates in reversing the progression of HCC, or express CD40/CD154 to directly stimulate the proliferation and metastasis of HCC cells, whereas PD-L1 expressed on monocytes combined with PD-1 on Breg cells lead to the exhaustion of Breg cell. (c) Macrophage expresses a higher level of PD-L1 resulting from incorporating exosomes derived from HCC, later interacting with PD-1 on CD8+T cell, and luring the apoptosis of CD8+T cell. And the same exosomes but different cargos trigger the polarization of the macrophage into the M2 subtype. (d) Exosomes containing CD86/AFP derived from DC activate naive T cells into

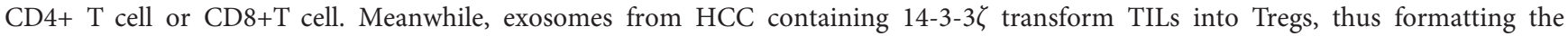
immunosuppressed environment of HCC.

other. Research showed that the therapeutic intervention of DC-TEXs can reduce Tregs and restore exhausted CD8 ${ }^{+}$ $\mathrm{T}$ cells in mice, thereby enhancing the efficacy of sorafenib and reducing the incidence of drug resistance [134]. The immunotherapy that combines anti-PD-1 monoclonal antibodies with DEX could be supposed as a potential strategy to reshape the immune microenvironment of poorly immunogenic HCC in situ.

4.4. NK Cell. Natural killer cell (NK cell) as the most important cells in the natural immune system can take up tumor-derived exosomes to help tumor cells escape the immune surveillance [135]. CircUHRF1, secreted extracellularly by HCC cells, upregulates the expression of $\mathrm{T}$ cell immunoglobulin and mucin domain 3 (Tim-3) and inhibits the secretion of IFN- $\gamma$ and TNF- $\alpha$ derived from NK cells, thereby inducing immunosuppression through NK cell dysfunction [34]. NKG2D ligand has strong capacities to downregulate homologous receptors and diminish the cytotoxic function of immune cells such as NK and NKT [136]. However, hepatocytes infected with HBV can release exosomes with a viral nucleic acid to induce macrophages to express NKG2D ligand and then inhibit NK cells activation, which may shape the immunosuppressive microenvironment of HCC [137].

Exosomes are a new type of secretory pathway of heat shock proteins (HSPs), and stress-inducible HSPs are considered endogenous "danger signals," which can improve tumor immunogenicity [138]. HCC cells treated with resistant anticancer drugs such as carboplatin and irinotecan hydrochloride increase the secretion of exosomes carrying HSP60, HSP70, and HSP90. Exosomes carrying HSPs can effectively stimulate the killing effect of NK cells and the production of granzyme B [139]. Similarly, HCC-derived exosomes modified with MS-275 (an epigenetic drug) can significantly enhance the killing effect of NK cells on HCC cells by upregulating MICB and HSP70 [140].

4.5. $T$ Cell. $T$ cells can mediate the death of abnormal cells through specifically-released perforin and granzymes, or the Fas-FasL mechanism. Through a variety of mechanisms, exosomes in the TIME can affect subsets of T cells, including $\mathrm{CD}^{+}$and $\mathrm{CD}^{+}{ }^{+} \mathrm{T}$ cells. In this part, we will introduce how T cell subsets, including $\mathrm{CD}^{+}, \mathrm{CD} 8^{+}$, and $\mathrm{NKT}$, function in the TIME and how they are regulated by exosomes. 
4.5.1. $C D 8^{+} T$ Cell. We have long believed that $\mathrm{CD} 8^{+} \mathrm{T}$ cells can recognize and kill tumor cells. Unfortunately, most tumor-infiltrating $\mathrm{CD}^{+} \mathrm{T}$ cells lowly express cell surface molecules CD28 and CD27 and highly express immunosuppressive factors Tim-3 and PD-1 [141]. This change causes the functional exhaustion of $\mathrm{CD}^{+} \mathrm{T}$ cells and loss of antitumor effect. At the same time, exosomes derived from a variety of cells in the tumor microenvironment can regulate the loss of antitumor effects of CD8 ${ }^{+} \mathrm{T}$ cells. Both LOXL4 and miR-23a-3p derived from HCC cells can be delivered by exosomes and activate the expression of PD-L1 in hepatic macrophages after the exosomal internalization, thereby further inhibiting the function of $\mathrm{CD}^{+} \mathrm{T}$ cells or promoting their apoptosis $[125,142]$.

As NKG2D receptors are expressed on $\mathrm{CD}^{+} \mathrm{T}$ cells, NKG2D ligands and ULBP carried by exosomes can inhibit the cytotoxicity of $\mathrm{CD}^{+} \mathrm{T}$ cells through NKG2D receptors and thereby induce HCC immune escape $[6,136]$. In contrast, DC cell-derived exosomes containing tumor antigens can effectively stimulate the transition of naive $\mathrm{T}$ cells into specific $\mathrm{CD} 8^{+} \mathrm{T}$ cells, maximizing the stimulation of MHCI restricted cytotoxicity against HCC [143]. It can be seen that exosomes in the tumor microenvironment have a dual regulatory effect on the function of $\mathrm{CD}^{+}$ T cells.

4.5.2. $\mathrm{CD}^{+} \mathrm{T}$ Cell. Although $\mathrm{CD} 4^{+} \mathrm{T}$ cells cannot directly kill tumor cells, $\mathrm{CD} 4^{+} \mathrm{T}$ cells can play an indirect role in the TIME. Evidence confirmed that HCC-derived exosomes are able to be endocytosed by $\mathrm{CD}^{+} \mathrm{T}$ cells, upregulating the inhibitory genes in $\mathrm{CD}^{+} \mathrm{T}$ cells, then leading to the loss of CD69 on their surface and decline of the function [144]. In addition, coculture of $\mathrm{CD} 4^{+} \mathrm{T}$ cells with HCC-derived exosomes can increase the expression level of immunosuppressive factors including IL-10, COX-2, TGF- $\beta$, and CTLA- 4 to inhibit the immune response to HCC [145].

4.5.3. Regulatory $T$ Cell. Regulatory $\mathrm{T}$ cell is a special type of $\mathrm{CD}^{+}{ }^{+} \mathrm{T}$ cell that highly expresses CD25 and specifically expresses forkhead box protein P3 (Foxp3) and can maintain immune homeostasis and immune tolerance through cell contact mechanisms or secretion of suppressive cytokines such as IL-10 and TGF- $\beta$ [146]. Tregs inhibit the secretion of IFN- $\gamma$, granzyme A, granzyme B, and perforin by NK cells and $\mathrm{CD} 8^{+} \mathrm{T}$ cells, thereby suppressing the antitumor effects. And Tregs are involved in the formation of the immunosuppressive microenvironment in HCC through their immunosuppressive effects [147]. Interestingly, exosomes of nasopharyngeal carcinoma origin promote the proliferation of $\mathrm{CD} 4^{+} \mathrm{CD} 25^{-} \mathrm{T}$ cells and their conversion to $\mathrm{CD} 4^{+} \mathrm{CD} 25^{+}$ regulatory T cells [148]. Subsequently, increased secretion of IL- 10 and TGF- $\beta$ mediates peripheral immune tolerance and immune escape of nasopharyngeal carcinoma. Similarly, Wang et al. found that HCC cells can produce 14-3-3 $\zeta$ protein, which can be delivered to tumor-infiltrating $\mathrm{T}$ lymphocytes (TILs) via exosomes [149]. They then demonstrated that exosomal $14-3-3 \zeta$ mediates the transition of TIL from effector T cells to Tregs. The above evidence supports that exosomes transmit critical signals during the transition of T cells to Tregs.

4.5.4. NKT Cell. NKT cells are a special T cell subset with characteristics of both NK cells and traditional T cells, accounting for $1 / 3$ of liver lymphocytes. Studies have revealed that exosomes released from adipose tissue-derived mesenchymal stem cells can promote the proliferation of NKT cells and give them a stronger anti-HCC effect [150].

4.6. B Cell. Breg cells are a type of B lymphocytes with immunomodulatory properties, which exert immunosuppressive effects mainly by producing cytokines such as TGF- $\beta$, IL-10, and IL-35. Similar to Tregs, Breg cells can also promote the progression of HCC through immune suppression. Shao et al. revealed for the first time that Berg cells can directly interact with HCC cells through the CD40/CD154 signaling pathway and thereby promote the proliferation, migration, and invasion of HCC cells [151].

A special type of Breg cells expression has been reported to be functionally activated after contact with monocytes expressing PD-L1. These PD-1high Breg cells facilitate the progression of HCC by mediating the dysfunction of cytotoxic $\mathrm{T}$ cells through the IL-10 pathway. In addition, ordinary B cells in tumor tissues can convert into Breg cells, which depends on exosomal HMGB1 released from HCC cells [106]. The resulting Breg cells can release a large amount of IL-10 and then inhibit the function of CD8+ T, thereby mediating the HCC immune escape. As for the mechanism of how other types of B cells and Breg cells exert immunomodulation in HCC, it needs to be further studied.

\section{Conclusion and Perspectives}

Exosomes of different origins loaded with various components such as miRNA, lncRNA, circRNA, DNA, protein, or other cargos correlate with angiogenesis, chemotherapy sensitivity, and metastasis of HCC. The complex crosstalk exists among immune cells, tumor cells, tumor-associated fibroblasts, and endothelial cells in TIME, in which exosomes are the vital mediators of these interactions. In particular, exosomes, which exist in the intercellular matrix of tumor tissues, serve as a bridge for various cells in the TIME to communicate with each other. Therefore, exosomes isolated directly from tissues can more directly reflect the molecular components affecting the TIME. Single-cell sequencing is a powerful weapon to analyze the TIME and can reveal the heterogeneity and dynamic changes of tumorinfiltrating immune cells and their potential role in immunotherapy. Combining the tissue-derived exosomes with single-cell sequencing technology can help to depict a complete image of the immune microenvironment in HCC. Although progress has been made in this field, the standardized method for the extraction of HCC tissue-derived exosomes has not been established, and the separation efficiency has not been guaranteed. As a whole, this combined 
technology has good application prospects worthy of further exploration.

A comprehensive understanding of the TIME will facilitate the development of new therapeutic targets. Recent studies support the dual role of exosomes in TIME of HCC. On the one hand, exosomes help to promote the polarization and proliferation of Tregs and M2 macrophages, thus contributing to building an immunosuppressive microenvironment in HCC. On the other hand, exosomes can promote CTL to kill HCC cells and exert antitumor effects, as shown in Figure 4. Currently, studies supported that dendritic cell-derived exosomes can load tumor-associated antigens, play the role of antigen presentation, and then activate the immune system to recognize and kill tumors. Moreover, multiple clinical trials have also shown exciting efficacy in using them as antitumor agents [152]. In the near future, the discovery and identification of exosomes that exert immune-activating or immunosuppressive effects will be the key basis for the development of specific, targeted therapeutics drugs.

In addition, in-depth exploration of exosomes in TIME is helpful to resist drug resistance in HCC. Although there are several options of therapeutic agents, acquired drug resistance is a major obstacle to the prognosis of HCC [153]. As previously noted, exosomal miRNAs are key molecules in regulating chemotherapy resistance; therefore, the role of exosomal pathway in obtaining drug resistance phenotype of HCC cannot be ignored. Regorafenib, with a broad range of kinase inhibitory effects, has shown good efficacy in HCC, which can inhibit angiogenesis and cell proliferation and also promote antitumor immunity [154]. However, the recent study proved that exosomes produced by HCC stem cells in a RAB27A-dependent manner can be ingested by differentiated tumor cells and then upregulate the expression of their intracellular drug resistance-associated gene Nanog, thereby increasing the resistance of HCC cells to Regorafenib [155]. Next, by knocking out the RAB27 A gene, the production of exosomes of HCC stem cells could be inhibited. Furthermore, the ability of these exosomes to increase the drug resistance was significantly reduced. Clearly, blocking exosomal pathway-mediated drug resistance signaling transduction is becoming a potential therapeutic strategy against the development of drug resistance in HCC.
Abbreviations
HCC: Hepatocellular carcinoma
TIME: Tumor immune microenvironment
ILV: ntraluminal vesicles
ESCRT: Endosomal sorting complexes required for transport
cfDNA: Cell-free DNA
EMT: Epithelial-to-mesenchymal transition
DEX: DC-derived exosomes
Tim-3: T cell immunoglobulin and mucin domain 3
ACD: Accidental cell death
RCD: $\quad$ Regulated cell death
AFP: Alpha-fetoprotein

HSP: Heat shock protein

TGF- $\beta$ : $\quad$ Transforming growth factor $\beta$

$\operatorname{IFN} \beta / \gamma$ : Interferon $\beta / \gamma$

ER Endoplasmic reticulum stress

stress:

DC: Dendritic cell

TILs: Tumor-infiltrating T lymphocytes

NK cell: Natural killer cell

Tregs: Regulatory T cells.

\section{Data Availability}

Not applicable.

\section{Ethical Approval}

Not applicable.

\section{Consent}

Not applicable.

\section{Conflicts of Interest}

The authors declare that they have no conflicts of interest.

\section{Authors' Contributions}

HYW and CXJ designed and revised the manuscript. CXJ, $\mathrm{CH}, \mathrm{PR}$, and WY drafted the manuscript. CXJ and ZXZ prepared the figures. All authors read and approved the final manuscript.

\section{Acknowledgments}

This work was supported by a grant from the Project of Science and Technology Department of Sichuan Province (2020JDTD0036).

\section{References}

[1] X. Duan, H. Li, M. Wang et al., "PSMC2/ITGA6 axis plays critical role in the development and progression of hepatocellular carcinoma," Cell Death Discovery, vol. 7, no. 1, Article ID 217, 2021.

[2] A. Osipov, M. T. Saung, L. Zheng, and A. G. Murphy, "Small molecule immunomodulation: the tumor microenvironment and overcoming immune escape," Journal for ImmunoTherapy of Cancer, vol. 7, no. 1, Article ID 224, 2019.

[3] A. S. Jadli, N. Ballasy, P. Edalat, and V. B. Patel, "Inside (sight) of tiny communicator: exosome biogenesis, secretion, and uptake," Molecular and Cellular Biochemistry, vol. 467, no. 1-2, pp. 77-94, 2020.

[4] R. Kalluri and V. S. LeBleu, "The biology, function, and biomedical applications of exosomes," Science (New York, N.Y.), vol. 367, no. 6478, 2020.

[5] J. P. Nolan and E. Duggan, "Analysis of individual extracellular vesicles by flow cytometry," Flow Cytometry Protocols, vol. 1678, pp. 79-92, 2018.

[6] L. Zhang and D. Yu, "Exosomes in cancer development, metastasis, and immunity," Biochimica et Biophysica Acta 
(BBA) - Reviews on Cancer, vol. 1871, no. 2, pp. 455-468, 2019.

[7] J. Kowal, G. Arras, M. Colombo et al., "Proteomic comparison defines novel markers to characterize heterogeneous populations of extracellular vesicle subtypes," Proceedings of the National Academy of Sciences, vol. 113, no. 8, pp. E968-E977, 2016.

[8] S. Nair, K. D. Tang, L. Kenny, and C. Punyadeera, "Salivary exosomes as potential biomarkers in cancer," Oral Oncology, vol. 84, pp. 31-40, 2018.

[9] J. M. Street, E. H. Koritzinsky, D. M. Glispie, R. A. Star, and P. S. T. Yuen, "Urine exosomes," Advances in Clinical Chemistry, vol. 78, pp. 103-122, 2017.

[10] H. Peinado, M. Alečković, S. Lavotshkin et al., "Melanoma exosomes educate bone marrow progenitor cells toward a pro-metastatic phenotype through MET," Nature Medicine, vol. 18, no. 6, pp. 883-891, 2012.

[11] X. Zhang, X. Yuan, H. Shi, L. Wu, H. Qian, and W. Xu, "Exosomes in cancer: small particle, big player," Journal of Hematology \& Oncology, vol. 8, no. 1, 83 pages, 2015.

[12] C. Lässer, M. Eldh, and J. Lötvall, "Isolation and characterization of RNA-containing exosomes," Journal of Visualized Experiments : Journal of Visualized Experiments, vol. 59, Article ID e3037, 2012.

[13] X. Huang, T. Yuan, M. Tschannen et al., "Characterization of human plasma-derived exosomal RNAs by deep sequencing," BMC Genomics, vol. 14, no. 1, Article ID 319, 2013.

[14] M. Tkach and C. Théry, "Communication by extracellular vesicles: where we are and where we need to go," Cell, vol. 164, no. 6, pp. 1226-1232, 2016.

[15] Y. Wang, K. Yin, J. Tian, X. Xia, J. Ma, and X. Tang, "Granulocytic myeloid-derived suppressor cells promote the stemness of colorectal cancer cells through exosomal S100A9," Advanced Science, vol. 6, no. 18, 2019.

[16] E. G. Trams, C. J. Lauter, J. Norman Salem Jr., and U. Heine, "Exfoliation of membrane ecto-enzymes in the form of micro-vesicles," Biochimica et Biophysica Acta (BBA) Biomembranes, vol. 645, no. 1, pp. 63-70, 1981.

[17] J. Huotari and A. Helenius, "Endosome maturation," The EMBO Journal, vol. 30, no. 17, pp. 3481-3500, 2011.

[18] M. Babst, D. J. Katzmann, W. B. Snyder, B. Wendland, and S. D. Emr, "Endosome-associated complex, ESCRT-II, recruits transport machinery for protein sorting at the multivesicular body," Developmental Cell, vol. 3, no. 2, pp. 283-289, 2002.

[19] D. J. Katzmann, M. Babst, and S. D. Emr, "Ubiquitin-dependent sorting into the multivesicular body pathway requires the function of a conserved endosomal protein sorting complex, ESCRT-I," Cell, vol. 106, no. 2, pp. 145-155, 2001.

[20] P. I. Hanson and A. Cashikar, "Multivesicular body morphogenesis," Annual Review of Cell and Developmental Biology, vol. 28, no. 1, pp. 337-362, 2012.

[21] T. Wollert, C. Wunder, J. Lippincott-Schwartz, and J. H. Hurley, "Membrane scission by the ESCRT-III complex," Nature, vol. 458, no. 7235, pp. 172-177, 2009.

[22] D. G. Meckes and N. Raab-Traub, "Microvesicles and viral infection," Journal of Virology, vol. 85, no. 24, pp. 12844-12854, 2011.

[23] B. György, T. G. Szabó, M. Pásztói et al., "Membrane vesicles, current state-of-the-art: emerging role of extracellular vesicles," Cellular and Molecular Life Sciences: CM, vol. 68, no. 16, pp. 2667-2688, 2011.
[24] K. Trajkovic, C. Hsu, S. Chiantia et al., "Ceramide triggers budding of exosome vesicles into multivesicular endosomes," Science, vol. 319, no. 5867, pp. 1244-1247, 2008.

[25] G. van Niel, S. Charrin, S. Simoes, M. Romao, L. Rochin, and P. Saftig, "The tetraspanin CD63 regulates ESCRT-independent and -dependent endosomal sorting during melanogenesis," Developmental Cell, vol. 21, no. 4, pp. 708-721, 2011.

[26] D. Wei, W. Zhan, Y. Gao et al., "RAB31 marks and controls an ESCRT-independent exosome pathway," Cell Research, vol. 31, no. 2, pp. 157-177, 2020.

[27] G. van Niel, G. D’Angelo, and G. Raposo, "Shedding light on the cell biology of extracellular vesicles," Nature Reviews Molecular Cell Biology, vol. 19, no. 4, pp. 213-228, 2018.

[28] W. Li, X. Ding, S. Wang et al., "Downregulation of serum exosomal miR-320d predicts poor prognosis in hepatocellular carcinoma," Journal of Clinical Laboratory Analysis, vol. 34, no. 6, Article ID e23239, 2020.

[29] L. Gramantieri, M. Baglioni, F. Fornari et al., "LncRNAs as novel players in hepatocellular carcinoma recurrence," Oncotarget, vol. 9, no. 80, pp. 35085-35099, 2018.

[30] Y. Luo, F. Liu, and R. Gui, "High expression of circulating exosomal circAKT3 is associated with higher recurrence in HCC patients undergoing surgical treatment," Surgical Oncology, vol. 33, pp. 276-281, 2020.

[31] G. Wang, W. Liu, Y. Zou et al., "Three isoforms of exosomal circPTGR1 promote hepatocellular carcinoma metastasis via the miR449a-MET pathway," EBioMedicine, vol. 40, pp. 432-445, 2019.

[32] S. Wang, Y. Yang, L. Sun, G. Qiao, Y. Song, and B. Liu, "Exosomal MicroRNAs as liquid biopsy biomarkers in hepatocellular carcinoma," OncoTargets and Therapy, vol. 13, pp. 2021-2030, 2020.

[33] J. Wang, J. Pu, Y. Zhang et al., "Exosome-transmitted long non-coding RNA SENP3-EIF4A1 suppresses the progression of hepatocellular carcinoma," Aging, vol. 12, no. 12, pp. 11550-11567, 2020.

[34] P. F. Zhang, C. Gao, X. Y. Huang et al., "Cancer cell-derived exosomal circUHRF1 induces natural killer cell exhaustion and may cause resistance to anti-PD1 therapy in hepatocellular carcinoma," Molecular Cancer, vol. 19, no. 1, Article ID 110, 2020.

[35] M. Haemmerle, R. L. Stone, D. G. Menter, V. AfsharKharghan, and A. K. Sood, "The platelet lifeline to cancer: challenges and opportunities," Cancer Cell, vol. 33, no. 6, pp. 965-983, 2018.

[36] X.-P. Tian, C.-Y. Wang, X.-H. Jin et al., "Acidic microenvironment up-regulates exosomal miR-21 and miR-10b in early-stage hepatocellular carcinoma to promote cancer cell proliferation and metastasis," Theranostics, vol. 9, no. 7, pp. 1965-1979, 2019.

[37] A. Sorop, R. Iacob, S. Iacob, D. Constantinescu, L. Chitoiu, and T. E. Fertig, "Plasma small extracellular vesicles derived miR-21-5p and miR-92a-3p as potential biomarkers for hepatocellular carcinoma screening," Frontiers in Genetics, vol. 11, 2020.

[38] L. Sun, Y. Su, X. Liu et al., "Serum and exosome long non coding RNAs as potential biomarkers for hepatocellular carcinoma," Journal of Cancer, vol. 9, no. 15, pp. 2631-2639, 2018.

[39] A. N. Muhammad Yusuf, R. A. Raja Ali, K. N. Muhammad Nawawi, and N. M. Mokhtar, "Potential biomarkers in NASH-induced liver cirrhosis with hepatocellular carcinoma: a preliminary work on roles of exosomal miR-182, 
miR-301a, and miR-373," Malaysian Journal of Pathology, vol. 42, no. 3, pp. 377-384, 2020.

[40] X. Ge, L. Tang, Y. Wang et al., "The diagnostic value of exosomal miRNAs in human bile of malignant biliary obstructions," Digestive and Liver Disease, vol. 53, no. 6, pp. 760-765, 2021.

[41] R. Crescitelli, C. Lässer, S. C. Jang et al., "Subpopulations of extracellular vesicles from human metastatic melanoma tissue identified by quantitative proteomics after optimized isolation," Journal of Extracellular Vesicles, vol. 9, no. 1, Article ID 1722433, 2020.

[42] Y. H. Park, H. W. Shin, A. R. Jung et al., "Prostate-specific extracellular vesicles as a novel biomarker in human prostate cancer," Scientific Reports, vol. 6, no. 1, Article ID 30386, 2016.

[43] L. J. Vella, B. J. Scicluna, L. Cheng et al., "A rigorous method to enrich for exosomes from brain tissue," Journal of Extracellular Vesicles, vol. 6, no. 1, Article ID 1348885, 2017.

[44] R. Crescitelli, C. Lässer, and J. Lötvall, "Isolation and characterization of extracellular vesicle subpopulations from tissues," Nature Protocols, vol. 16, no. 3, pp. 1548-1580, 2021.

[45] C. Kahlert, S. A. Melo, A. Protopopov et al., "Identification of double-stranded genomic DNA spanning all chromosomes with mutated KRAS and p53 DNA in the serum exosomes of patients with pancreatic cancer," Journal of Biological Chemistry, vol. 289, no. 7, pp. 3869-3875, 2014.

[46] V. Bernard, D. U. Kim, F. A. San Lucas et al., "Circulating nucleic acids are associated with outcomes of patients with pancreatic cancer," Gastroenterology, vol. 156, no. 1, pp. 108-118, 2019.

[47] C. Kahlert, "Liquid biopsy: is there an advantage to analyzing circulating exosomal DNA compared to cfDNA or are they the same?" Cancer Research, vol. 79, no. 10, pp. 2462-2465, 2019.

[48] L. Yan, Y. Chen, J. Zhou, H. Zhao, H. Zhang, and G. Wang, "Diagnostic value of circulating cell-free DNA levels for hepatocellular carcinoma," International Journal of Infectious Diseases, vol. 67, pp. 92-97, 2018.

[49] K. Allenson, J. Castillo, F. A. San Lucas et al., "High prevalence of mutantKRAS in circulating exosome-derived DNA from early-stage pancreatic cancer patients," Annals of Oncology, vol. 28, no. 4, pp. 741-747, 2017.

[50] H. Xu, X. Dong, Y. Chen, and X. Wang, "Serum exosomal hnRNPH1 mRNA as a novel marker for hepatocellular carcinoma," Clinical Chemistry and Laboratory Medicine, vol. 56, no. 3, pp. 479-484, 2018.

[51] A. Abd El Gwad, M. Matboli, A. El-Tawdi et al., "Role of exosomal competing endogenous RNA in patients with hepatocellular carcinoma," Journal of Cellular Biochemistry, vol. 119, no. 10, pp. 8600-8610, 2018.

[52] G. Helmlinger, F. Yuan, M. Dellian, and R. K. Jain, "Interstitial $\mathrm{pH}$ and $\mathrm{pO} 2$ gradients in solid tumors in vivo: high-resolution measurements reveal a lack of correlation," Nature Medicine, vol. 3, no. 2, pp. 177-182, 1997.

[53] R. A. Cairns, I. Papandreou, P. D. Sutphin, and N. C. Denko, "Metabolic targeting of hypoxia and HIF1 in solid tumors can enhance cytotoxic chemotherapy," Proceedings of the National Academy of Sciences, vol. 104, no. 22, pp. 9445-9450, 2007.

[54] J. Folkman, "Tumor angiogenesis: therapeutic implications," New England Journal of Medicine, vol. 285, no. 21, pp. 1182-1186, 1971.

[55] Y. Matsuura, H. Wada, H. Eguchi et al., "Exosomal miR-155 derived from hepatocellular carcinoma cells under hypoxia promotes angiogenesis in endothelial cells," Digestive Diseases and Sciences, vol. 64, no. 3, pp. 792-802, 2018.

[56] W. Chen, L. Huang, J. Liang, Y. Ye, S. He, and J. Niu, "Hepatocellular carcinoma cells-derived exosomal microRNA-378b enhances hepatocellular carcinoma angiogenesis," Life Sciences, vol. 273, Article ID 119184, 2021.

[57] K. Hu, N. F. Li, J. R. Li, Z. G. Chen, J. H. Wang, and L. Q. Sheng, "Exosome circCMTM3 promotes angiogenesis and tumorigenesis of hepatocellular carcinoma through miR-3619-5p/SOX9," Hepatology Research, vol. 51, no. 11, pp. 1139-1152, 2021.

[58] S.-S. Dong, D.-D. Dong, Z.-F. Yang et al., "Exosomal miR3682-3p suppresses angiogenesis by targeting ANGPT1 via the RAS-MEK1/2-ERK1/2 pathway in hepatocellular carcinoma," Frontiers in Cell and Developmental Biology, vol. 9, Article ID 633358, 2021.

[59] K. Takahashi, I. K. Yan, H. Haga, and T. Patel, "Modulation of hypoxia-signaling pathways by extracellular linc-RoR," Journal of Cell Science, vol. 127, no. Pt 7, pp. 1585-1594, 2014.

[60] A. Kumar and G. Deep, "Hypoxia in tumor microenvironment regulates exosome biogenesis: molecular mechanisms and translational opportunities," Cancer Letters, vol. 479, pp. 23-30, 2020.

[61] Y. Yu, Z. Min, z Zhou Zhihang et al., "Hypoxia-induced exosomes promote hepatocellular carcinoma proliferation and metastasis via miR-1273f transfer," Experimental Cell Research, vol. 385, no. 1, Article ID 111649, 2019.

[62] F. B. Pallasch and U. Schumacher, "Angiotensin inhibition, TGF- $\beta$ and EMT in cancer," Cancers, vol. 12, no. 10, 2020.

[63] Q. L. Chen, C. F. Xie, K. L. Feng et al., "microRNAs carried by exosomes promote epithelial-mesenchymal transition and metastasis of liver cancer cells," American Journal of Tourism Research, vol. 12, no. 10, pp. 6811-6826, 2020.

[64] Y. Xu, L. Cao, G. Chen et al., "Human umbilical cord mesenchymal stem cells-derived exosomal microRNA-451a represses epithelial-mesenchymal transition of hepatocellular carcinoma cells by inhibiting ADAM10," RNA Biology, vol. 18, no. 10, pp. 1-16, 2020.

[65] L. Chen, P. Guo, Y. He et al., "HCC-derived exosomes elicit HCC progression and recurrence by epithelial-mesenchymal transition through MAPK/ERK signalling pathway," Cell Death \& Disease, vol. 9, no. 5, Article ID 513, 2018.

[66] Z. Qu, J. Feng, H. Pan, Y. Jiang, Y. Duan, and Z. Fa, "Exosomes derived from HCC cells with different invasion characteristics mediated EMT through TGF- $\beta /$ Smad signaling pathway," OncoTargets and Therapy, vol. 12, pp. 6897-6905, 2019.

[67] B. Bakir, A. M. Chiarella, J. R. Pitarresi, and A. K. Rustgi, "EMT, MET, plasticity, and tumor metastasis," Trends in Cell Biology, vol. 30, no. 10, pp. 764-776, 2020.

[68] Z. Tang, J. He, J. Zou, S. Yu, X. Sun, and L. Qin, "Cisplatinresistant HepG2 cell-derived exosomes transfer cisplatin resistance to cisplatin-sensitive cells in HCC," PeerJ, vol. 9, Article ID e11200, 2021.

[69] H. Zhang, T. Deng, R. Liu et al., "CAF secreted miR-522 suppresses ferroptosis and promotes acquired chemo-resistance in gastric cancer," Molecular Cancer, vol. 19, no. 1, p. $43,2020$.

[70] T. Liu, X. Zhang, L. Du et al., "Exosome-transmitted miR128-3p increase chemosensitivity of oxaliplatin-resistant colorectal cancer," Molecular Cancer, vol. 18, no. 1, p. 43, 2019.

[71] G. Lou, L. Chen, C. Xia et al., "MiR-199a-modified exosomes from adipose tissue-derived mesenchymal stem cells 
improve hepatocellular carcinoma chemosensitivity through mTOR pathway," Journal of Experimental \& Clinical Cancer Research: Climate Research, vol. 39, no. 1, p. 4, 2020.

[72] G. Lou, X. Song, F. Yang et al., "Exosomes derived from miR-122-modified adipose tissue-derived MSCs increase chemosensitivity of hepatocellular carcinoma," Journal of Hematology \& Oncology, vol. 8, no. 1, Article ID 122, 2015.

[73] G. Wang, W. Zhao, H. Wang et al., "Exosomal MiR-744 inhibits proliferation and sorafenib chemoresistance in hepatocellular carcinoma by targeting PAX2," Medical Science Monitor, vol. 25, pp. 7209-7217, 2019.

[74] M. J. Shang, C. Jiang, C. Y. Tu, X. L. Lv, J. D. Zhu, and C. X Shao, "Exosomes function as nanoparticles to transfer miR-199a-3p to reverse chemoresistance to cisplatin in hepatocellular carcinoma," Bioscience Reports, vol. 40, no. 7, 2020.

[75] Y. Liu, J. Tan, S. Ou, J. Chen, and L. Chen, "Adipose-derived exosomes deliver miR-23a/b to regulate tumor growth in hepatocellular cancer by targeting the VHL/HIF axis," Journal of Physiology \& Biochemistry, vol. 75, no. 3, pp. 391-401, 2019.

[76] H. Xia, L. L. P. J. Ooi, and K. M. Hui, "MicroRNA-216a/217induced epithelial-mesenchymal transition targets PTEN and SMAD7 to promote drug resistance and recurrence of liver cancer," Hepatology, vol. 58, no. 2, pp. 629-641, 2013.

[77] K. Liu, S. Liu, W. Zhang et al., "miR-494 promotes cell proliferation, migration and invasion, and increased sorafenib resistance in hepatocellular carcinoma by targeting PTEN," Oncology Reports, vol. 34, no. 2, pp. 1003-1010, 2015.

[78] X. Fu, M. Liu, S. Qu et al., "Exosomal microRNA-32-5p induces multidrug resistance in hepatocellular carcinoma via the PI3K/Akt pathway," Journal of Experimental \& Clinical Cancer Research: Climate Research, vol. 37, no. 1, p. 52, 2018.

[79] D. Tang, R. Kang, T. V. Berghe, P. Vandenabeele, and G. Kroemer, "The molecular machinery of regulated cell death," Cell Research, vol. 29, no. 5, pp. 347-364, 2019.

[80] A. Strasser and D. L. Vaux, "Cell death in the origin and treatment of cancer," Molecular Cell, vol. 78, no. 6, pp. 1045-1054, 2020.

[81] D. Xue, J. Han, Y. Liu, H. Tuo, and Y. Peng, "Current perspectives on exosomes in the diagnosis and treatment of hepatocellular carcinoma (review)," Cancer Biology \& Therapy, vol. 22, no. 4, pp. 279-290, 2021.

[82] R. Tang, J. Xu, B. Zhang et al., "Ferroptosis, necroptosis, and pyroptosis in anticancer immunity," Journal of Hematology \& Oncology, vol. 13, no. 1, Article ID 110, 2020.

[83] L. Q. Cao, X. W. Yang, Y. B. Chen, D. W. Zhang, X. F. Jiang, and P. Xue, "Exosomal miR-21 regulates the TETs/PTENp1/ PTEN pathway to promote hepatocellular carcinoma growth," Molecular Cancer, vol. 18, no. 1, Article ID 148, 2019.

[84] F. Aredia and A. I. Scovassi, "A new function for miRNAs as regulators of autophagy," Future Medicinal Chemistry, vol. 9, no. 1, pp. 25-36, 2017.

[85] M. J. Czaja, "Functions of autophagy in hepatic and pancreatic physiology and disease," Gastroenterology, vol. 140, no. 7, pp. 1895-1908, 2011.

[86] Y. Lee and B. Jang, "The role of autophagy in hepatocellular carcinoma," International Journal of Molecular Sciences, vol. 16, no. 11, pp. 26629-26643, 2015.

[87] N. Wang, H.-Y. Tan, S. Li, and Y. Feng, "Atg9b deficiency suppresses autophagy and potentiates endoplasmic reticulum stress-associated hepatocyte apoptosis in hepatocarcinogenesis," Theranostics, vol. 7, no. 8, pp. 2325-2338, 2017.
[88] H. Yu, P. Guo, X. Xie, Y. Wang, and G. Chen, "Ferroptosis, a new form of cell death, and its relationships with tumourous diseases," Journal of Cellular and Molecular Medicine, vol. 21, no. 4, pp. 648-657, 2017.

[89] J. Du, Z. Wan, C. Wang et al., "Designer exosomes for targeted and efficient ferroptosis induction in cancer via chemo-photodynamic therapy," Theranostics, vol. 11, no. 17, pp. 8185-8196, 2021.

[90] N. Ren, T. Jiang, C. Wang et al., "LncRNA ADAMTS9-AS2 inhibits gastric cancer (GC) development and sensitizes chemoresistant GC cells to cisplatin by regulating miR-223-3p/NLRP3 axis," Aging, vol. 12, no. 11, pp. 11025-11041, 2020.

[91] K. P. O’Brien, S. Khan, K. E. Gilligan, H. Zafar, P. Lalor, and C. Glynn, "Employing mesenchymal stem cells to support tumor-targeted delivery of extracellular vesicle (EV)-encapsulated microRNA-379," Oncogene, vol. 37, no. 16, pp. 2137-2149, 2018.

[92] H. Zhang, T. Deng, S. Ge et al., "Exosome circRNA secreted from adipocytes promotes the growth of hepatocellular carcinoma by targeting deubiquitination-related USP7," Oncogene, vol. 38, no. 15, pp. 2844-2859, 2019.

[93] Y. Su, X. Lv, W. Yin et al., "CircRNA Cdrlas functions as a competitive endogenous RNA to promote hepatocellular carcinoma progression," Aging, vol. 11, no. 19, pp. 8183-8203, 2019.

[94] Y. Lin, Z.-H. Zheng, J.-X. Wang, Z. Zhao, and T.-Y. Peng, "Tumor cell-derived exosomal circ-0072088 suppresses migration and invasion of hepatic carcinoma cells through regulating MMP-16," Frontiers in Cell and Developmental Biology, vol. 9, Article ID 726323, 2021.

[95] X. Huang, L. Sun, S. Wen et al., "RNA sequencing of plasma exosomes revealed novel functional long noncoding RNAs in hepatocellular carcinoma," Cancer Science, vol. 111, no. 9, pp. 3338-3349, 2020.

[96] H. Gu, C. Yan, H. Wan et al., "Mesenchymal stem cellderived exosomes block malignant behaviors of hepatocellular carcinoma stem cells through a lncRNA C5orf66-AS1/ microRNA-127-3p/DUSP1/ERK axis," Human Cell, vol. 34, no. 6, pp. 1812-1829, 2021.

[97] L.-p. Wang, J. Lin, X.-q. Ma et al., "Exosomal DLX6-AS1 from hepatocellular carcinoma cells induces M2 macrophage polarization to promote migration and invasion in hepatocellular carcinoma through microRNA-15a-5p/CXCL17 axis," Journal of Experimental \& Clinical Cancer Research, vol. 40, no. 1, Article ID 177, 2021.

[98] C. Zhu, Y. Su, L. Liu, S. Wang, Y. Liu, and J. Wu, "Circular RNA hsa_circ_0004277 stimulates malignant phenotype of hepatocellular carcinoma and epithelial-mesenchymal transition of peripheral cells," Frontiers in Cell and Developmental Biology, vol. 8, 2021.

[99] X. Peng, X. Li, S. Yang, M. Huang, S. Wei, and Y. Ma, "LINC00511 drives invasive behavior in hepatocellular carcinoma by regulating exosome secretion and invadopodia formation," Journal of Experimental \& Clinical Cancer Research, vol. 40, no. 1, 2021.

[100] L. Yang, X. Peng, Y. Li et al., "Long non-coding RNA HOTAIR promotes exosome secretion by regulating RAB35 and SNAP23 in hepatocellular carcinoma," Molecular Cancer, vol. 18, no. 1, p. 78, 2019.

[101] L. Lyu, W. Yang, J. Yao et al., "The diagnostic value of plasma exosomal hsa_circ_0070396 for hepatocellular carcinoma," Biomarkers in Medicine, vol. 15, no. 5, pp. 359-371, 2021. 
[102] P. Lyu, Z. Zhai, Z. Hao, H. Zhang, and J. He, "CircWHSC1 serves as an oncogene to promote hepatocellular carcinoma progression," European Journal of Clinical Investigation, vol. 51, no. 6, Article ID e13487, 2021.

[103] Y. Wang, R. Gao, J. Li et al., "Downregulation of hsa_circ_ 0074854 suppresses the migration and invasion in hepatocellular carcinoma via interacting with $\mathrm{HuR}$ and via suppressing exosomes-mediated macrophage M2 polarization," International Journal of Nanomedicine, vol. 16, pp. 2803-2818, 2021.

[104] Z. Cheng, Z. Lei, P. Yang et al., "Exosome-transmitted p120catenin suppresses hepatocellular carcinoma progression via STAT3 pathways," Molecular Carcinogenesis, vol. 58, no. 8, pp. 1389-1399, 2019.

[105] H. Sun, C. Wang, B. Hu et al., "Exosomal S100A4 derived from highly metastatic hepatocellular carcinoma cells promotes metastasis by activating STAT3," Signal Transduction and Targeted Therapy, vol. 6, no. 1, Article ID 187, 2021.

[106] L. Ye, Q. Zhang, Y. Cheng et al., “Tumor-derived exosomal HMGB1 fosters hepatocellular carcinoma immune evasion by promoting TIM-1+ regulatory B cell expansion," Journal for ImmunoTherapy of Cancer, vol. 6, no. 1, Article ID 145, 2018.

[107] M. Lin, W. Liao, M. Dong et al., "Exosomal neutral sphingomyelinase 1 suppresses hepatocellular carcinoma via decreasing the ratio of sphingomyelin/ceramide," FEBS Journal, vol. 285, no. 20, pp. 3835-3848, 2018.

[108] K. Jiang, C. Dong, Z. Yin et al., "Exosome-derived ENO1 regulates integrin $\alpha 6 \beta 4$ expression and promotes hepatocellular carcinoma growth and metastasis," Cell Death \& Disease, vol. 11, no. 11, Article ID 972, 2020.

[109] N. Milman, L. Ginini, and Z. Gil, "Exosomes and their role in tumorigenesis and anticancer drug resistance," Drug Resistance Updates, vol. 45, pp. 1-12, 2019.

[110] H. Zhao, L. Yang, J. Baddour et al., "Tumor microenvironment derived exosomes pleiotropically modulate cancer cell metabolism," Elife, vol. 5, Article ID e10250, 2016.

[111] J. I. Sanchez, J. Jiao, S.-Y. Kwan et al., "Lipidomic profiles of plasma exosomes identify candidate biomarkers for early detection of hepatocellular carcinoma in patients with cirrhosis," Cancer Prevention Research, vol. 14, no. 10, pp. 955-962, 2021.

[112] S. Finkin, D. Yuan, I. Stein et al., "Ectopic lymphoid structures function as microniches for tumor progenitor cells in hepatocellular carcinoma," Nature Immunology, vol. 16, no. 12, pp. 1235-1244, 2015.

[113] J. L. Messina, D. A. Fenstermacher, S. Eschrich et al., "12-Chemokine gene signature identifies lymph node-like structures in melanoma: potential for patient selection for immunotherapy?" Scientific Reports, vol. 2, no. 1, Article ID 765, 2012.

[114] B. Hurt, R. Schulick, B. Edil, K. C. El Kasmi, and C. Barnett, "Cancer-promoting mechanisms of tumor-associated neutrophils," The American Journal of Surgery, vol. 214, no. 5, pp. 938-944, 2017.

[115] R. V. Sionov, Z. G. Fridlender, and Z. Granot, "The multifaceted roles neutrophils play in the tumor microenvironment," Cancer Microenvironment, vol. 8, no. 3, pp. 125-158, 2014.

[116] C. Haider, J. Hnat, R. Wagner et al., "Transforming growth factor- $\beta$ and Axl induce CXCL5 and neutrophil recruitment in hepatocellular carcinoma," Hepatology, vol. 69, no. 1, pp. 222-236, 2019.
[117] C. Hernandez, P. Huebener, J.-P. Pradere, D. J. Antoine, R. A. Friedman, and R. F. Schwabe, "HMGB1 links chronic liver injury to progenitor responses and hepatocarcinogenesis," Journal of Clinical Investigation, vol. 128, no. 6, pp. 2436-2451, 2018.

[118] X. Zhang, H. Shi, X. Yuan, P. Jiang, H. Qian, and W. Xu, "Tumor-derived exosomes induce N2 polarization of neutrophils to promote gastric cancer cell migration," Molecular Cancer, vol. 17, no. 1, Article ID 146, 2018.

[119] D. F. Quail and J. A. Joyce, "Microenvironmental regulation of tumor progression and metastasis," Nature Medicine, vol. 19, no. 11, pp. 1423-1437, 2013.

[120] L. Wang, Y. Y. Hu, J. L. Zhao et al., "Targeted delivery of miR-99b reprograms tumor-associated macrophage phenotype leading to tumor regression," Journal for ImmunoTherapy of Cancer, vol. 8, no. 2, 2020.

[121] M. Shen, Y. Shen, X. Fan, R. Men, T. Ye, and L. Yang, "Roles of macrophages and exosomes in liver diseases," Frontiers of Medicine, vol. 7, Article ID 583691, 2020.

[122] X. Li, Y. Lei, M. Wu, and N. Li, "Regulation of macrophage activation and polarization by HCC-derived exosomal lncRNA TUC339," International Journal of Molecular Sciences, vol. 19, no. 10, 2018.

[123] C. Yin, Q. Han, D. Xu, B. Zheng, X. Zhao, and J. Zhang, "SALL4-mediated upregulation of exosomal miR-146a-5p drives T-cell exhaustion by M2 tumor-associated macrophages in HCC," OncoImmunology, vol. 8, no. 7, Article ID 1601479, 2019.

[124] J. R. Cubillos-Ruiz, S. E. Bettigole, and L. H. Glimcher, "Tumorigenic and immunosuppressive effects of endoplasmic reticulum stress in cancer," Cell, vol. 168, no. 4, pp. 692-706, 2017.

[125] J. Liu, L. Fan, H. Yu, J. Zhang, Y. He, and D. Feng, "Endoplasmic reticulum stress causes liver cancer cells to release exosomal miR-23a-3p and up-regulate programmed death ligand 1 expression in macrophages," Hepatology, vol. 70, no. 1, pp. 241-258, 2019.

[126] G. Liu, X. Ouyang, Y. Sun et al., "The miR-92a-2-5p in exosomes from macrophages increases liver cancer cells invasion via altering the AR/PHLPP/p-AKT/ $\beta$-catenin signaling," Cell Death \& Differentiation, vol. 27, no. 12, pp. 3258-3272, 2020.

[127] J. Wu, W. Gao, Q. Tang et al., "Retracted: M2 macrophagederived exosomes facilitate HCC metastasis by transferring $\alpha$ M $\beta 2$ integrin to tumor cells," Hepatology, vol. 73, no. 4, pp. 1365-1380, 2021.

[128] C. Wei, X. Yang, N. Liu et al., "Tumor microenvironment regulation by the endoplasmic reticulum stress transmission mediator golgi protein 73 in mice," Hepatology, vol. 70, no. 3, pp. 851-870, 2019.

[129] X. Zhong, Y. Zhou, Y. Cao et al., "Enhanced antitumor efficacy through microwave ablation combined with a dendritic cell-derived exosome vaccine in hepatocellular carcinoma," International Journal of Hyperthermia, vol. 37, no. 1, pp. 1210-1218, 2020.

[130] N. El-Sayed, E. Korotchenko, S. Scheiblhofer, R. Weiss, and M. Schneider, "Functionalized multifunctional nanovaccine for targeting dendritic cells and modulation of immune response," International Journal of Pharmaceutics, vol. 593, Article ID 120123, 2021.

[131] Z. Lu, B. Zuo, R. Jing et al., "Dendritic cell-derived exosomes elicit tumor regression in autochthonous hepatocellular carcinoma mouse models," Journal of Hepatology, vol. 67, no. 4, pp. 739-748, 2017. 
[132] S. Yu, C. Liu, K. Su et al., "Tumor exosomes inhibit differentiation of bone marrow dendritic cells," The Journal of Immunology, vol. 178, no. 11, pp. 6867-6875, 2007.

[133] A. H. Lau and A. W. Thomson, "Dendritic cells and immune regulation in the liver," Gut, vol. 52, no. 2, pp. 307-314, 2003.

[134] S. Shi, Q. Rao, C. Zhang, X. Zhang, Y. Qin, and Z. Niu, "Dendritic cells pulsed with exosomes in combination with PD-1 antibody increase the efficacy of sorafenib in hepatocellular carcinoma model," Translational Oncology, vol. 11, no. 2, pp. 250-258, 2018.

[135] T. Huyan, Y. Du, Q. Huang, Q. Huang, and Q. Li, "Uptake characterization of tumor cell-derived exosomes by natural killer cells," Iranian Journal of Public Health (English ed.), vol. 47, no. 6, pp. 803-813, 2018.

[136] L. Mincheva-Nilsson and V. Baranov, "Cancer exosomes and NKG2D receptor-ligand interactions: impairing NKG2Dmediated cytotoxicity and anti-tumour immune surveillance," Seminars in Cancer Biology, vol. 28, pp. 24-30, 2014.

[137] T. Kouwaki, Y. Fukushima, T. Daito et al., "Extracellular vesicles including exosomes regulate innate immune responses to hepatitis B virus infection," Frontiers in Immunology, vol. 7, Article ID 335, 2016.

[138] L. Elsner, V. Muppala, M. Gehrmann et al., "The heat shock protein HSP70 promotes mouse NK cell activity against tumors that express inducible NKG2D ligands," The Journal of Immunology, vol. 179, no. 8, pp. 5523-5533, 2007.

[139] L.-H. Lv, Y.-L. Wan, Y. Lin et al., "Anticancer drugs cause release of exosomes with heat shock proteins from human hepatocellular carcinoma cells that elicit effective natural killer cell antitumor responses in vitro," Journal of Biological Chemistry, vol. 287, no. 19, pp. 15874-15885, 2012.

[140] W. Xiao, W. Dong, C. Zhang et al., "Effects of the epigenetic drug MS-275 on the release and function of exosome-related immune molecules in hepatocellular carcinoma cells," European Journal of Medical Research, vol. 18, no. 1, p. 61, 2013.

[141] J. Crespo, H. Sun, T. H. Welling, Z. Tian, and W. Zou, "T cell anergy, exhaustion, senescence, and stemness in the tumor microenvironment," Current Opinion in Immunology, vol. 25, no. 2, pp. 214-221, 2013.

[142] H. Y. Tan, N. Wang, C. Zhang, Y. T. Chan, M. F. Yuen, and Y. Feng, "Lysyl oxidase-like 4 fosters an immunosuppressive microenvironment during hepatocarcinogenesis," Hepatology, vol. 73, no. 6, pp. 2326-2341, 2021.

[143] J. Li, S. Huang, Z. Zhou et al., "Exosomes derived from rAAV/AFP-transfected dendritic cells elicit specific T cellmediated immune responses against hepatocellular carcinoma," Cancer Management and Research, vol. 10, pp. 4945-4957, 2018.

[144] L. Muller, M. Mitsuhashi, P. Simms, W. E. Gooding, and T. L. Whiteside, "Tumor-derived exosomes regulate expression of immune function-related genes in human $\mathrm{T}$ cell subsets," Scientific Reports, vol. 6, no. 1, Article ID 20254, 2016.

[145] L. Muller, P. Simms, C. S. Hong et al., "Human tumorderived exosomes (TEX) regulate Treg functions via cell surface signaling rather than uptake mechanisms," OncoImmunology, vol. 6, no. 8, Article ID e1261243, 2017.

[146] M. Walecki, F. Eisel, J. Klug et al., "Androgen receptor modulates Foxp3 expression in CD4+CD25+Foxp3+ regulatory T-cells," Molecular Biology of the Cell, vol. 26, no. 15, pp. 2845-2857, 2015.

[147] A. Granito, L. Muratori, C. Lalanne et al., "Hepatocellular carcinoma in viral and autoimmune liver diseases: role of CD4+ CD25+ Foxp3+ regulatory $\mathrm{T}$ cells in the immune microenvironment," World Journal of Gastroenterology, vol. 27, no. 22, pp. 2994-3009, 2021.

[148] D. Mrizak, N. Martin, C. Barjon et al., "Effect of nasopharyngeal carcinoma-derived exosomes on human regulatory T cells," Journal of the National Cancer Institute, vol. 107, no. 1, Article ID 363, 2015.

[149] X. Wang, H. Shen, G. Zhangyuan et al., "14-3-3 ל delivered by hepatocellular carcinoma-derived exosomes impaired antitumor function of tumor-infiltrating Tlymphocytes," Cell Death \& Disease, vol. 9, no. 2, Article ID 159, 2018.

[150] S.-F. Ko, H.-K. Yip, Y.-Y. Zhen et al., "Adipose-derived mesenchymal stem cell exosomes suppress hepatocellular carcinoma growth in a rat model: apparent diffusion coefficient, natural killer T-cell responses, and histopathological features," Stem Cells International, vol. 2015, pp. 1-11, 2015.

[151] Y. Shao, C. M. Lo, C. C. Ling et al., "Regulatory B cells accelerate hepatocellular carcinoma progression via CD40/ CD154 signaling pathway," Cancer Letters, vol. 355, no. 2, pp. 264-272, 2014.

[152] Y. Yao, C. Fu, L. Zhou, Q. S. Mi, and A. Jiang, "DC-derived exosomes for cancer immunotherapy," Cancers, vol. 13, no. $15,2021$.

[153] J. M. Llovet, R. Montal, D. Sia, and R. S. Finn, "Molecular therapies and precision medicine for hepatocellular carcinoma," Nature Reviews Clinical Oncology, vol. 15, no. 10, pp. 599-616, 2018.

[154] A. Granito, A. Forgione, S. Marinelli et al., "Experience with regorafenib in the treatment of hepatocellular carcinoma," Therapeutic Advances in Gastroenterology, vol. 14, Article ID 17562848211016959, 2021.

[155] H. Huang, J. Hou, K. Liu, Q. Liu, L. Shen, and B. Liu, "RAB27A-dependent release of exosomes by liver cancer stem cells induces Nanog expression in their differentiated progenies and confers regorafenib resistance," Journal of Gastroenterology and Hepatology, vol. 36, no. 12, 2021. 